\title{
Primacy-recency and the sequential presentation of attitudinal stimuli'
}

DONN BYRNE AND OLIVER LONDON

UNIVERSITY OF TEXAS
The present investigation dealt with the effect of the sequential presentation of positive and negative attitudinal stimuli on impression formation. There were four experimental groups of $10 \mathrm{Ss}$ each: complete similarity to S's attitudes, complete dissimilarity, and two $50 \%$ similarity conditions with a progression from similarity to dissimilarity and the reverse. The four conditions were found to differ significantly ( $p<.001)$, but the difference between the two 50\% groups was not significant. Neither a primacy nor a recency effect was found.

A variable of continuing research interest has been the sequence in which stimuli with positive and negative qualities are presented to the S. Asch (1946) found that the initial adjectives in a list have the greatest influence on the resulting impression; this primacy effect has been replicated in subsequent work (e.g., Anderson \& Barrios, 1961). Asch proposed a Gestalt interpretation, but a more quantitative and convincing explanation was offered by Anderson \& Hubert (1963), based on differential attention. It was proposed that Ss pay a decreasing degree of attention to each item as the list progresses. Thus, primacy effects disappear when attention is controlled by informing Ss that recall will be required afterward (Anderson \& Hubert, 1963), by reducing the list to two adjectives (Anderson \& Barrios, 1961), or by requiring the $S$ to respond after each item (Stewart, 1965).

A second type of stimulus variable is that of positive or negative evaluations from the stimulus person directed at the $\mathrm{S}$. In a series of interactions and evaluations, strong recency effects are found (Aronson \& Linder, 1965; Berkowitz, 1960a, b).

Still a third type of stimulus information consists of attitude statements which are similar to or dissimilar from the views of the $S$. As the proportion of similar attitudes increases, attraction increases (e.g., Byrne \& Nelson, 1964). In these studies, the sequence of similar and dissimilar statements has not been investigated but simply controlled through randomization. Unfortunately, the adjective trait investigations and the personal evaluation studies lead to opposite predictions with respect to primacy versus recency effects. The effect of the sequential presentation of positive and negative attitudinal stimuli is explored in this experiment. Method

Four experimental conditions were established: similar, dissimilar, similar-dissimilar, and dissimilarsimilar. The Ss were 40 University of Texas students who had taken a 56-item attitude scale early in the semester. They were randomly assigned to one of the conditions, with equal numbers of males and females in each. A special tape recording was prepared for each S; a same-sex stranger verbalized a pre-arranged response to each of the 56 items. In the Similar (S) condition, all responses agreed with those of the $S$, and in the Dissimilar (D) condition all responses disagreed. In the Similar-Dissimilar (S-D) condition, in blocks of eight items the following numbers of similar and dissimilar responses were given: $8-0,7-1,6-2$, $4-4,2-6,1-7,0-8$. In the Dissimilar-Similar (D-S) condition, the S-D pattern was simply reversed.

The Ss were told that the experiment involved the accuracy of interpersonal judgment and that the task was to determine how much one individual could learn about another on the basis of a tape recording. After hearing the recording, the S evaluated the stranger on the sixitem Interpersonal Judgment Scale. The last two items (liking and working together) are summed to yield the measure of the dependent variable.

\section{Results}

A simple one-way analysis of variance yielded highly significant differences among the groups $(F=14.58$, $\mathrm{df}=3 / 36, \mathrm{p}<.001$ ). The critical comparison, however, is between the S-D and D-S groups. The difference between them was not statistically significant $(t=1.1$, $\mathrm{df}=18$, N.S.).

\section{Discussion}

How is it possible to reconcile the present results with those of previous investigations? The recency effects of the personal evaluation studies have been interpreted by Aronson \& Linder (1965) in terms of a gain-loss hypothesis. Briefly, the success or failure obtained in the interpersonal interactions adds to or subtracts from the effects of the evaluative statements themselves. Since interaction is crucial to this formulation, it should not be applicable to the present data.

The present findings are compatible with those of the adjective trait studies if one assumption can be made: because of their greater saliency to the $S$, their variety of content, their relative familiarity, and the relative independence of each topic, attitude statements sequentially exposed do not yield a decreasing attention function.

There is another aspect of the impression-formation problem which is compatible with the present findings. Adjective traits may be treated quantitatively in terms of scaled favorableness and a weighting coefficient. Anderson $(1965 a, b)$ presents a weighted average model in which response to a given set of adjectives is a 


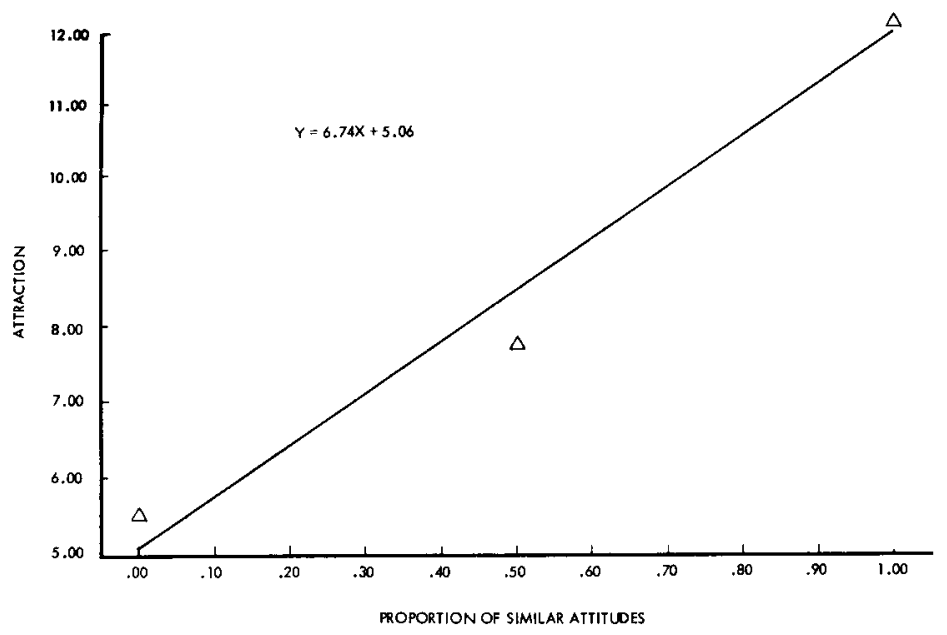

Fig. 1. Attraction as a linear function of proportion of similar attitudes: correspondence between present data and prediction based on the Byme-Clore formula.

direct function of the average multiplicative value of favorableness times weight. In the interpersonal attraction studies, it is precisely this same kind of model which describes the S's response to a series of attitude statements as a positive linear function of proportion of similar attitudes (e.g., Byrne \& Nelson, 1965) with equal weights assigned to each attitude item (Byrne \& Nelson, 1964). Under conditions comparable to the present research, Byrne \& Clore (1966) found that the linear function between proportion of similar attitudes and attraction is described by the formula $Y=6.74 X+5.06$. In Fig. 1 , the Byrne-Clore function is plotted along with the means of the $S, D$, and $S-D$ plus $D-S$ groups. There is obviously a close correspondence between curve and data. A goodness of fit analysis indicated a nonsignificant difference between predicted and observed values $\left(F=.76, d f=3 / 37, N_{.} . S_{.}\right)$. Such findings lend further support to Anderson's averaging model.

\section{References}

Anderson, N. H. Primacy effects in personality impression formation using a generalized order effect paradigm. J. Pers. soc. Psychol., 1965a, 2, 1-9,

Anderson, N. H. Averaging versus adding as a stimulus-combination rule in impression formation. J. exp. Psychol., 1965b, 70, 394400.

Anderson, N. H., \& Barrios, A. A. Primacy effects in personality impression formation. J. abnorm. soc. Psychol., 1961, 63, 346350 .
Anderson, N. H., \& Hubert, S. Effects of concomitant verbal recall on order effects in personality impression formation. J. verbal Learn. verbal Behav., 1963, 2, 379-391.

Aronson, E., \& Linder, D. Gain and loss of esteem as determinants of interpersonal attractiveness. J. exp. soc. Psychol., 1965, 1, 156-171.

Asch, S. E. Forming impressions of personality. J. abnorm. soc. Psychol., 1946, 41, 258-290.

Berkowitz, L. Some factors affecting the reduction of overt hostility. J. abnorm. soc. Psychol., 1960a, 60, 14-21.

Berkowitz, L. Repeated frustrations and expectations in hostility atousal. J. abnorm. soc. Psychol., 1960b, 60, 422-429.

Byrne, D., \& Clore, G. L., Jr. Predicting interpersonal attraction toward strangers presented in three different stimulus modes. Psychon. Sci., 1966, 4, 239-240.

Byme, D., \& Nelson, D. Attraction as a function of attikude similarity-dissimilarity: the effect of topic importance. Psychon. Sci., 1964, 1, 93-94.

Byme, D., \& Nelson, D. Attraction as a linear function of proportion of positive reinforcements. J. Pers. soc. Psychol., 1965, $1,659-663$.

Stewart, R. H. Effect of continuous responding on the order effect in personality impression formation. J. Pers. soc. Psychol., $1965,1,161-165$.

\section{Note}

1. This research was supported in part by Research Grant MH11178-01 from the National Institute of Mental Health, United States Public Health Service. 